This is a postprint version of the following published document:

Óscar Fernández ; Álvaro Gómez ; Ángel Vegas ; Gregorio J. Molina-Cuberos ; Ismael Barba. Diode switchable chiral metamaterial structure for polarization manipulation. In: 2017 IEEE MTT-S International Conference on Numerical Electromagnetic and Multiphysics Modeling and Optimization for RF, Microwave, and Terahertz Applications (NEMO)

DOI: https://doi.org/10.1109/NEMO.2017.7964226

(C) 2017 IEEE. Personal use of this material is permitted. Permission from IEEE must be obtained for all other uses, in any current or future media, including reprinting/republishing this material for advertising or promotional purposes, creating new collective works, for resale or redistribution to servers or lists, or reuse of any copyrighted component of this work in other works. 


\section{Diode Switchable Chiral Metamaterial Structure for Polarization Manipulation}

\author{
Oscar Fernández, Álvaro Gómez \\ and Angel Vegas \\ Dpto. Ingeniería de Comunicaciones \\ Universidad de Cantabria \\ E39005 Santander, Spain \\ fernanos@unican.es, \\ gomezal@unican.es, \\ vegasa@unican.es
}

\author{
Gregorio J. Molina-Cuberos \\ Dpto. Electromagnetismo y \\ Electrónica \\ Universidad de Murcia \\ E30100 Murcia, Spain \\ gregomc@um.es
}

\author{
Ismael Barba \\ Dpto. Electricidad y Electrónica \\ Universidad de Valladolid \\ E47011 Valladolid, Spain \\ ibarba@ee.uva.es
}

\begin{abstract}
This communication presents a novel diode switchable chiral metamaterial structure that manipulates the polarization in different ways depending on the active bias lines. Three different bias states that provide three totally different behaviors have been considered: polarization rotator, circular polarization converter and linear to circular converter.
\end{abstract}

Keywords-Chiral metamaterial; diode; switchable

\section{INTRODUCTION}

Planar metamaterials are artificial structures constituted by metallic inclusions periodically distributed on a substrate. The electromagnetic properties of this kind of media come from the pattern and the geometric distribution of the inclusions rather than their components properties.

Tunable metamaterials are structures capable of changing its electromagnetic properties through the aid of external stimuli. These stimulations can be used to modify the conductivity of semiconducting substrates [1], alter geometric parameters in microelectromechanical systems [2], or bias semiconductor based diodes embedded into the metamaterial unit cells [3].

Focusing our attention on diode tunable metamaterials, in the literature it can be found several works focused on taking advance of the reflection features of these media: absorbers with semiconductors [4]-[5], impedance surface [6] or polarization modulation [7]. However, few available works take advantage of the tunability in transmission [8].

In this communication, a switchable chiral metamaterial (CMM) structure constituted by a bilayered CMM [9] with embedded diodes joining in series each unit cell with its neighbors, Fig. 1, is presented. Modifying the bias voltage, the diodes state and thus the connections with neighboring cells are changed. Consequently, different geometrical patterns can be obtained depending on the applied bias voltage. Along this work, three bias states of the schematic of Fig. 1 are analyzed. In each state, the bias lines are switched on or off in order to modify the polarization of an incident wave in these ways: as a polarization rotator, as a circular polarization converter and as a linear to circular polarization converter.

This work has been partially supported by the Spanish Government MINECO through the ERDF co-funded Research Projects TEC2014-55463C3-1-P, TEC2014-55463-C3-2-P and TEC2014-55463-C3-3-P.

\section{Diode Switchable Chiral Metamaterial}

Fig. 1 shows the pattern of each side of the proposed structure formed by the CMM and the PIN diodes. On each face, all the rows (columns) of diodes share the same bias line. As stated in [4], the diodes of the rows (columns) are turned on when the applied bias voltage is greater than the sum of their threshold voltages. In this forward biased state, the diode is modelled as a short circuit. Therefore, the unit cells of the rows (columns) are electrically connected. If no bias voltage is applied, the diode acts as an open circuit.

The switchable CMM has been implemented in Rogers RO4003C, with dielectric constant $\varepsilon_{r}=3.55$, $\tan \delta=0.0027$, thickness $d=1.52 \mathrm{~mm}$ and a copper cladding of $35 \mu \mathrm{m}$. The geometrical parameters of the structure are displayed in the figure caption. For its numerical characterization, the transmission matrix for linearly polarized incident waves (1) have been obtained through numerical simulations using the Finite Differences Time Domain engine of the Keysight EMPro 3D EM software ${ }^{\circledR}$.

$$
\left(\begin{array}{l}
E_{x}^{\mathrm{tra}} \\
E_{y}^{\mathrm{tra}}
\end{array}\right)=T_{L P}\left(\begin{array}{l}
E_{x}^{\mathrm{inc}} \\
E_{y}^{\mathrm{inc}}
\end{array}\right)=\left(\begin{array}{ll}
t_{x x} & t_{x y} \\
t_{y x} & t_{y y}
\end{array}\right)\left(\begin{array}{l}
E_{x}^{\mathrm{inc}} \\
E_{y}^{\mathrm{inc}}
\end{array}\right) .
$$

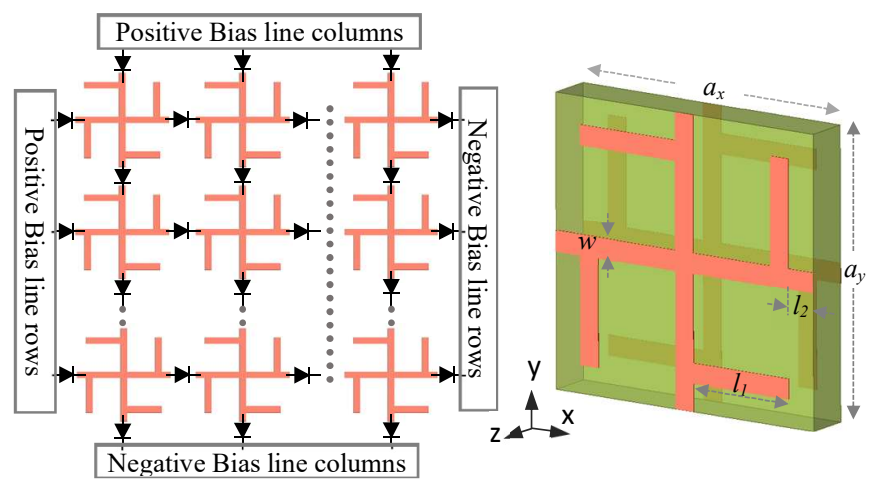

Fig. 1. Layout of each proposed structure side. Inset: Unit cell of the CMM structure. Dimensions (in mm): $l_{l}=3.7, l_{2}=0.95, w=0.7, a_{x}=a_{y}=10$. 
From this transmission matrix, $T_{L P}$, other related transmission matrices, linear to circular transmission matrix, $T_{C L}$, and circular transmission matrix, $T_{C}$, can be obtained [10]:

$$
\begin{gathered}
\boldsymbol{T}_{C L}=\left(\begin{array}{cc}
t_{+x} & t_{+y} \\
t_{-x} & t_{-y}
\end{array}\right)=\frac{1}{\sqrt{2}}\left(\begin{array}{cc}
t_{x x}+j t_{y x} & t_{x y}+j t_{y y} \\
t_{x x}-j t_{y x} & t_{x y}-j t_{y y}
\end{array}\right) \\
\boldsymbol{T}_{C}=\left(\begin{array}{ll}
t_{++} & t_{+-} \\
t_{-+} & t_{--}
\end{array}\right)=\frac{1}{2}\left(\begin{array}{ll}
t_{x x}+t_{y y}+j\left(t_{x y}-t_{y x}\right) & t_{x x}-t_{y y}-j\left(t_{x y}+t_{y x}\right) \\
t_{x x}-t_{y y}+j\left(t_{x y}+t_{y x}\right) & t_{x x}+t_{y y}-j\left(t_{x y}-t_{y x}\right)
\end{array}\right)
\end{gathered}
$$

Here, $t_{a b}$ represents the transmission coefficient of a wave with polarization $a$ when the impinging wave displays polarization $b$, wherein $a$ and $b$ may refer to $x$ or $y$ linear polarization or right$(\mathrm{RHCP},+)$ or left-handed (LHCP, - ) circular polarization.

\section{A. Polarization plane rotator}

The first analyzed case occurs when the bias lines are off. Under this conditions the unit cells are not electrically connected, resulting the well-known conjugated gammadion CMM [11], Fig. 2. This structure acts as polarization plane rotator providing pure rotation of $30^{\circ}$, Fig. 3 .
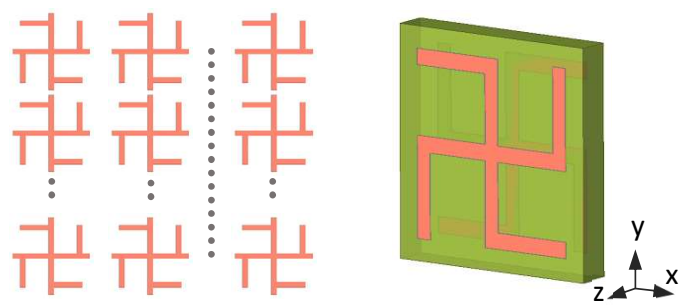

Fig. 2. Schematic of the pattern of each side of the Conjugated gammadion. Inset: Unit cell of the CMM structure.

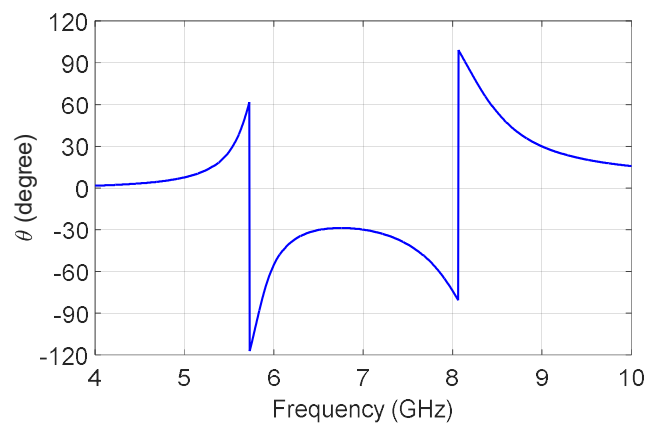

Fig. 3. Polarization plane rotation of the conjugated gammadion.

\section{B. Circular Polarization Converter}

Next, the bias voltage is applied to the columns bias line of each side of the metamaterial. Thus, the diodes embedded in the vertical wires (parallel to the $y$ axis) behaves as short circuits and the diodes of the horizontal wires act as open circuits. With this feed, the cells are interconnected by columns, resulting the structure of Fig. 4.

Fig. 5 shows the variation of the transmission coefficients versus frequency when impinging with a linearly polarized wave. Here, it can be observed that if the incident wave is $y$ polarized, the co-polar transmission is high in the entire band. However, when the incident wave is $x$-polarized, the co-polar transmission coefficient is lower than 0.5 except around 10.52 $\mathrm{GHz}$, wherein there is a peak of 0.8 . Meanwhile, both crosspolar transmission coefficients are very low, lower than 0.2 across the entire band.

Using (3), the transmission coefficients for circularly polarized incident waves are obtained (see Fig. 6). Here, it should be observed that, around $10.55 \mathrm{GHz}$, the co-polar transmission is very low $\left(\left|t_{-}\right|=0.3\right.$ and $\left.\left|t_{++}\right|=0.15\right)$ but the crosspolar transmission is very high, greater than 0.8 . Therefore, this structure behaves as a polarization converter, since it transforms the handedness of an incident circularly polarized wave into its opposite one.

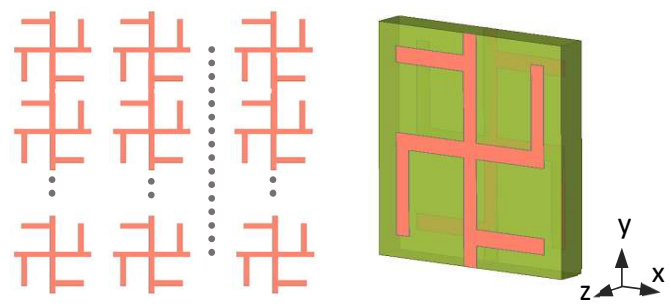

Fig. 4. Schematic of the pattern of each side of the Circular Polarization Converter. Inset: Unit cell of the CMM structure.

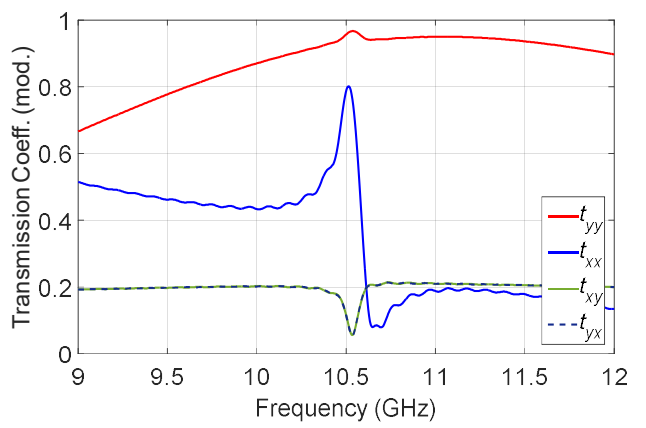

Fig. 5. Linear transmission coeffs. of the Circular polarization converter.

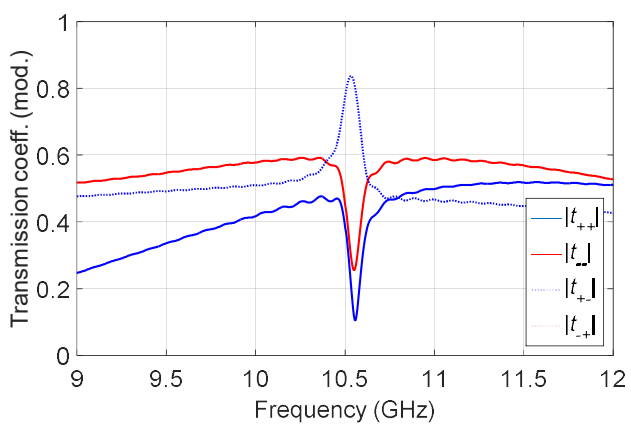

Fig. 6. Circular transmission coeffs. of the circular polarization converter.

The polarization conversion ratio $P C R_{ \pm}=\left|t_{ \pm \mp}\right|^{2} /\left(\left|t_{ \pm \mp}\right|^{2+}\left|t_{\mp \mp}\right|^{2}\right)$, i.e., the converter efficiency, is around $10.55 \mathrm{GHz}$ greater that $90 \%$ (Fig. 7). It is worth of mention that, at this frequency, the efficiency is greater when the handedness conversion is from RH to LH because $\left|t_{--}\right|>\left|t_{++}\right|$.

\section{Linear. to Circular Polarization Converter}

Finally, the diodes of the vertical wires are biased on one side of the substrate, whereas on the other face the biased diodes are the horizontal ones. This schematic, shown in Fig. 8, behaves as a linear to circular polarization converter. 


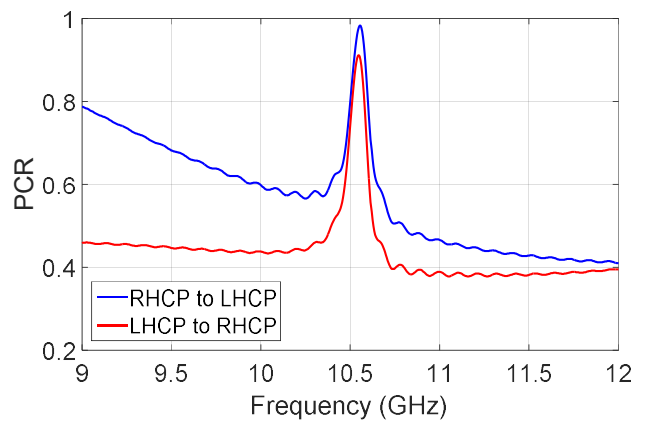

Fig. 7. Polarization conversion ratio of the circular polarization converter.

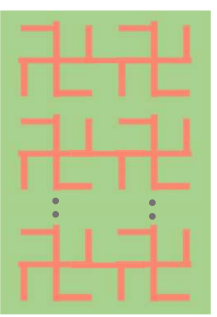

Side $+z$

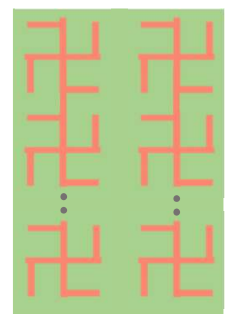

Side $-\mathrm{z}$

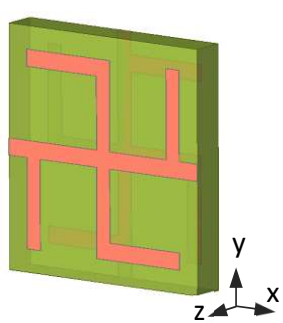

Fig. 8. Both sides of the Linear. to circular polarization converter. Inset Unit cell of the CMM structure.

Fig. 9 shows the linear to circular transmission coefficients as a function of frequency for two different operating bands. It can be verified that, when a linearly polarized wave impinges from $-z$ a left-handed circular polarized wave is transmitted. The performance in any operation band depends on the polarization direction of the incident wave. If it is perpendicular to the $y$-axis, the response occurs around at $5 \mathrm{GHz}$ (Fig. 9a) whereas in the case of polarization parallel to the $x$-axis, the response occurs around $13.5 \mathrm{GHz}$ (Fig. 9b). In both cases the LH component transmission reaches values higher than 0.9 .

Now, the polarization conversion efficiency is defined, similarly to (4), as the transmittance of the desired component to the total transmittance ratio, $\mathrm{PCR}_{ \pm x, y}=\left|t_{ \pm x, y}\right|^{2} /\left(\left|t_{+x, y}\right|^{2+}\left|t_{-x, y}\right|^{2}\right)$. Fig. 10 shows the polarization conversion ratio in the two frequency bands described previously. The conversion from linear to lefthanded circular polarization presents a PCR $>90 \%$ in a bandwidth greater than $1.3 \mathrm{GHz}$.

\section{CONCLUSIONS}

This communication presents a Chiral Metamaterial structure with embedded PIN diodes. This switchable structure manipulates the polarization in different ways depending on the active bias lines. Under these conditions, three different bias states have been considered which provides three totally different behaviors. As a polarization rotator (bias lines off) the structure has shown pure rotation of $30^{\circ}$ which corresponds to more than $840^{\circ} / \lambda$. When biasing only the diodes arranged in columns or rows on both faces, the structure works as a highly efficient polarization converter by transforming the handedness of an incident circularly polarized wave into its opposite one. Finally, the structure can also act as a wideband linear to circular converter if only one bias line is active per face and these bias lines are orthogonal.
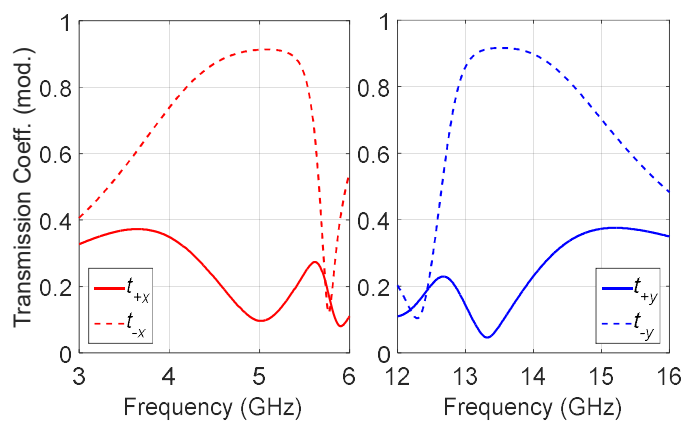

Fig. 9. Linear to Circular transmission coefficients of the linear to circular polarization converter

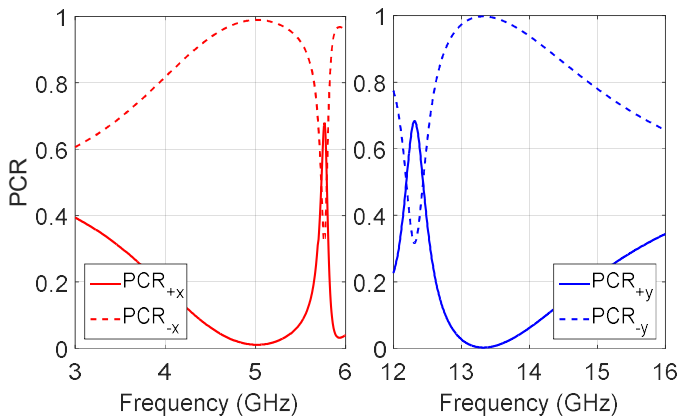

Fig. 10. Polarization conversion ratio of the linear to circular polarization converter.

\section{REFERENCES}

[1] B. B. Jin ; C. Li ; C. H. Zhang ; L. Kang ; W. W. Xu ; J. Chen ; P. H. Wu "High transmission superconducting $\mathrm{THz}$ switch based on a electrically tunable metamaterial," Infrared, Millimeter, and Terahertz waves (IRMMW-THz), 2016 41st International Conference on, 2016.

[2] H. Tao, A. C. Strikwerda, K. Fan, W. J. Padilla, X. Zhang, and R. D. Averitt, "MEMS based structurally tunable metamaterials at terahertz frequencies," J. Infrared Millim. Waves, vol. 32, no. 5, pp. 580-595, 2011.

[3] H.-T. Chen, W. J. Padilla, J. M. Zide, A. C. Gossard, A. J. Taylor, and R. D. Averitt, "Active terahertz metamaterial devices," Nature (London) 444, pp. 597-600, 2006.

[4] W. Xu and S. Sonkusale, "Microwave diode switchable metamaterial reflector/absorber," Applied Physics Letters 103, 031902, 2013.

[5] X. Xu, J. Jiang, L. Miao, Q. Chen, and B. Sun, "Design of tunable metamaterial absorbers based on PIN diodes," IEICE Electronics Express, vol. 9, no. 17, pp. 1408-1413, 2012.

[6] D.F., Sievenpiper, J. H. Schaffner, H. J. Song, R. Y. Loo and G. Tangonan, "Two-dimensional beam steering using an electrically tunable impedance surface," IEEE Trans. Antennas Propag., vol. 51, no. 10, pp. 2713-2722, 2003.

[7] B. Zhu, Y. Feng, J. Zhao, C. Huang, Z. Wang and T. Jiang, "Polarization modulation by tunable electromagnetic metamaterial reflector/absorber," Optics express, vol. 18, no 22, pp 13196-23203, 2010.

[8] I. V. Shadrivov,M. Lapine,Y. S. Kivshar, Nonlinear, Tunable and Active Metamaterials, Springer, 2015.

[9] O. Fernández, Á. Gómez, A. Vegas, G. J. Molina-Cuberos, and A. J. García-Collado, "Losses reduction in composite chiral metamaterials," The 36th PIERS in Prague, Czech Republic, 6-9, 2015.

[10] C. Menzel, C. Rockstuhl and F. Lederer, "Advanced Jones Calculus for the classification of periodic metamaterials," Phys. Rev. A, vol. 82, no. 5, pp. 053811, 2010

[11] R. Zhao, L. Zhang, J. Zhou, Th. Koschny, and C.M. Soukoulis, "Conjugated gammadion chiral metamaterial with uniaxial optical activity and negative refractive index," Phys. Rev. B, vol. 83, no. 1, pp. 035105, 2011. 